\title{
Submitted: High frequency ultrasound assessment of labial glands Accepted: 07.10.2020 \\ Published: simulating small nodules or granulomas after lip augmentation
} 18.12.2020

\author{
Ewa Skrzypek¹, Robert Krzysztof Mlosek² \\ ${ }^{1}$ Department of History of Medicine, Medical University of Warsaw, Poland \\ ${ }^{2}$ Ultrasound Diagnostic Department, Faculty of Medical Sciences, Medical University \\ of Warsaw, Warsaw, Poland \\ Correspondence: Ewa Skrzypek, MD, PhD, Department of History of Medicine, ul. Litewska 16, \\ 00-575 Warsaw, Poland; tel. 48604075 561, e-mail:ewa.skrzypek@wum.edu.pl
}

DOI: $10.15557 /$ JoU.2020.0046

\section{Keywords}

lips,

fillers,

complications, labial glands,

high-frequency ultrasound

\begin{abstract}
Aim of the study: The aim of this study is to evaluate the utility of high-frequency ultrasound in the assessment of the nature and differentiation of lumps occurring in the labial mucosa after lip filling procedures. Material and methods: The study sample included 5 women aged from 28 to 43 years (mean age: 37.4 years) who previously underwent a lip augmentation or rejuvenation procedure. Photographic documentation was taken and high-frequency ultrasound assessment was performed in all the patients. The presence of deposits, foreign body granulomas, fibrosis and labial glands was mainly investigated taking into account the shape of the abnormalities, their margins, surface area, location and echogenicity. In order to confirm the diagnosis, histopathological examination was performed. Results: The examinations performed made it possible to differentiate between granulomas and persistent deposit nodules and to demonstrate the presence of massive fibrosis and of labial glands pushed out by these abnormalities with the glands presenting as hypoechoic oval areas. Histopathological examination confirmed the morphology of labial glands reported by the patients as uncomfortable lumps felt from the side of the mucosa, which clinically simulate persistent deposit nodules or granulomas. Conclusions: High-frequency ultrasound is a method that is essential for the correct differentiation between complications of tissue filler procedures. This, in turn, makes it possible to apply the right treatment. In this study, it was demonstrated for the first time that the lumps reported by patients who have had a lip filling procedure may be the result of labial glands being pushed out by deposits, granulomas or massive fibrosis, which are complications of such procedures.
\end{abstract}

\section{Introduction}

Lips are a very important part of facial attractiveness. From time immemorial, mainly women have enhanced their lips as an indication of youth, beauty and sensuality ${ }^{(1,2)}$. Ideal lips are full with well-defined vermilion border. Passage of time and the associated subcutaneous adipose tissue atrophy, loss of elasticity, effects of gravity and the remodelling of the skeleton and muscles lead to the ageing of this area. Beginning in the third decade of life, internal factors such as hormonal and genetic changes have a negative effect on the quality and structure of the skin. External factors, including the impact of the environment, exposure to UV radiation or smoking result in a change of skin integrity.

For this reason, lip augmentation, and thus rejuvenation using fillers, in recent years mainly those based on hyaluronic acid (HA), has become a very fashionable and widely available procedure. When performing this procedure, one should always take into account the context of the whole perilabial area set by the base of the nose, 
the cheeks and the chin. From the aesthetic point of view, the right proportion between the upper lip and the lower lip is also important, which is $1: 1.6$ in young Caucasian women $^{(1,2)}$.

There is a rich market of products for reducing any signs of lip ageing, which include implants, botulinum toxin, laser treatments, micropigmentation and fillers, which are the most widely used and most popular solution. Fillers play an important role in lip and perilabial area rejuvenation.

Unfortunately, due to the lack of an ideal filling substance, a significant increase in the number of procedures and cases when they are performed by unauthorised individuals, there has been a growing number of complications.

The most common complications of lip filling procedures include nodules that are deposits of the filling material and foreign body granulomas (FBG). They are very difficult for clinical differentiation, especially since aesthetic medicine clinics offer a very limited range of tools for diagnosing complications.

High frequency ultrasound (HFUS) turns out to be the only effective and non-invasive method for diagnosing complications of lip filling procedures. It currently makes it possible to differentiate between deposits and granulomas, as well as any other doubtful cases in which diagnosis based on exclusively clinical evidence is simply impossible ${ }^{(3)}$.

The aim of this paper is to assess the utility of high-frequency ultrasound in the evaluation of clinical diagnosis of small nodules or granulomas after lip filling procedures. What is particularly important is that histopathological studies also confirmed the morphology of these abnormalities observed on ultrasound, similar to an earlier study on the differentiation between nodules and granulomas $^{(3)}$.

\section{Material and methods}

The study sample included 5 women aged from 28 to 43 years (mean age: 37.4 years) who sought medical help for a complication after a lip filling procedure: they complained of small lumps felt from the side of the mucosa of both lips, which were sometimes uncomfortable during lip movement. In all the patients, the changes were previously clinically diagnosed as nodules or granulomas.

Photographic documentation were taken and the lip mucosa was palpated. Detailed analysis of the study sample is presented in Tab. 1 .

Subsequently, ultrasound examination was performed using the Philips Epiq 5 ultrasound scanner (Philips, Bothel, USA) equipped with an L18-5 broadband linear transducer set to the lowest imaging depth and highest resolution and the DermaMed high-frequency ultrasound scanner (Dramiński S.A., Olsztyn, Poland) equipped with a $48 \mathrm{MHz}$ mechanical sector transducer.

Next, histopathological examination was performed of small nodules surgically removed from the side of the oral mucosa. The specimens were preserved in $4 \%$ buffered formalin, embedded in paraffin, cut into $4 \mu \mathrm{m}$ pieces and routinely stained with haematoxylin and eosin, and additionally with Alcian Blue pH 2.5 and Periodic Acid-Schiff (P.A.S).

\section{Results}

In patients complaining of small lumps felt from the side of the mucosa of both lips occurring after lip filling procedures, which were often uncomfortable to them during lip movement, clinical examination involving palpation confirmed their presence (Fig. 1).

In nearly all cases, ultrasound examination of the lips demonstrated filler deposits; in 3 cases, it showed hyaluronic acid deposits presenting as anechoic focal abnormalities,

Tab. 1. Characteristics of the study sample

\begin{tabular}{|c|c|c|c|c|c|c|}
\hline No. & Age at complication & Lump site & Filler type & $\begin{array}{c}\text { Time to } \\
\text { complication (years } \\
\text { from procedure) }\end{array}$ & Clinical diagnosis & $\begin{array}{l}\text { Ultrasound } \\
\text { diagnosis }\end{array}$ \\
\hline 1. & 38 & upper lip & unknown & 12 & $\begin{array}{l}\text { nodules or } \\
\text { granulomas }\end{array}$ & $\begin{array}{l}\text { massive fibrosis, } \\
\text { labial glands }\end{array}$ \\
\hline 2. & 40 & lower lip & HA a few times & 5 & $\begin{array}{l}\text { nodules or } \\
\text { granulomas }\end{array}$ & $\begin{array}{c}\text { massive fibrosis, } \\
\text { labial glands catching } \\
\text { on the incisors }\end{array}$ \\
\hline 3. & 28 & $\begin{array}{l}\text { lower lip, } \\
\text { upper lip }\end{array}$ & HA a few times & 4 & $\begin{array}{l}\text { nodules or } \\
\text { granulomas }\end{array}$ & $\begin{array}{l}\text { persistent deposits, } \\
\text { labial glands }\end{array}$ \\
\hline 4. & 43 & $\begin{array}{l}\text { upper lip, } \\
\text { lower lip }\end{array}$ & PAAG & 12 & $\begin{array}{l}\text { nodules or } \\
\text { granulomas }\end{array}$ & $\begin{array}{l}\text { granulomas, } \\
\text { persistent deposits, } \\
\text { labial glands }\end{array}$ \\
\hline 5. & 38 & $\begin{array}{l}\text { upper lip, } \\
\text { lower lip }\end{array}$ & HA twice & 1 & $\begin{array}{l}\text { nodules or } \\
\text { granulomas }\end{array}$ & $\begin{array}{c}\text { deposits, granulomas, } \\
\text { labial glands }\end{array}$ \\
\hline
\end{tabular}




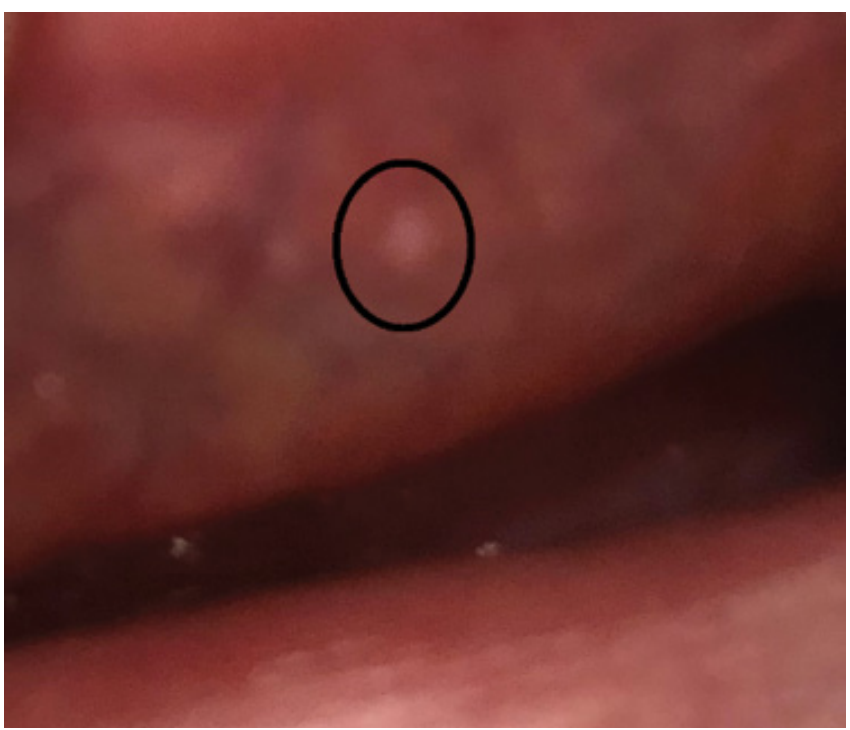

Fig. 1. Clinical image of a pushed out labial gland presenting as a small nodule of the upper lip

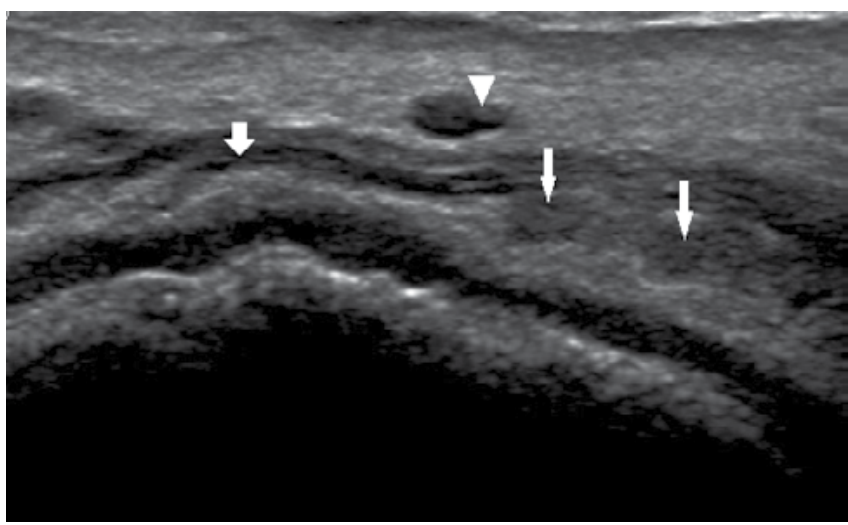

Fig. 2. Ultrasound image (Philips Epiq 5) of a hyaluronic acid deposit (arrow head) indenting the orbicularis oris (bold arrow) and labial glands (thin arrows)

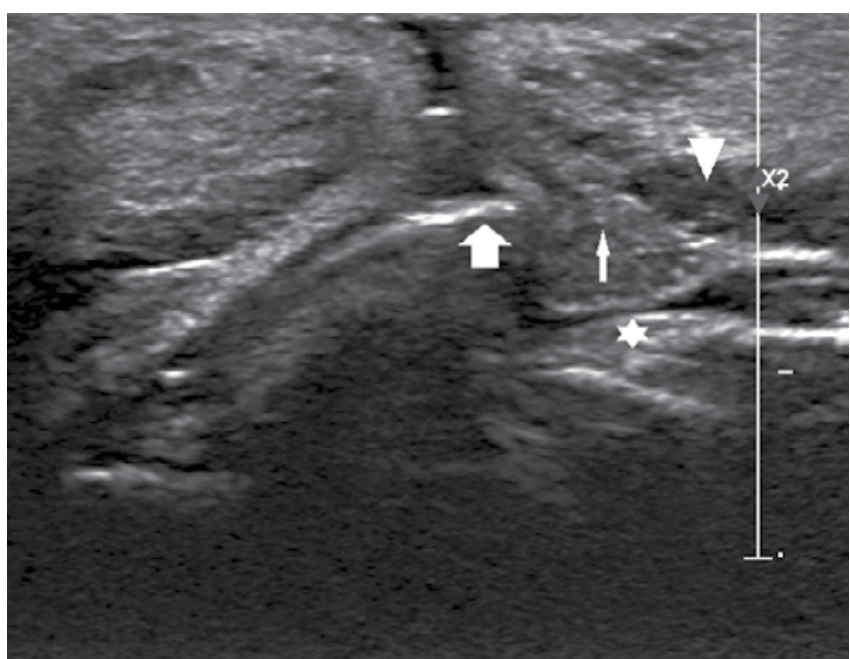

Fig. 3. Ultrasound image (Philips Epiq 5) of a hyaluronic acid deposit (arrow head) in the lower lip pushing out a labial gland (thin arrow) catching on the upper incisor (bold arrow); maxillary mucosa (asterisk) usually oval in shape, with sharp, regular external margins. They were usually accompanied with the indentation of the orbicularis oris muscle and minor salivary glands being pushed out, which are seen as hypoechoic, oval, welldefined areas located just below the oral mucosa on examination with the Epiq 5 machine (Fig. 2). In one case, the pushed out glands caught on the teeth, thus being at risk of irritation (Fig. 3).

The use of permanent fillers is usually complicated by granulomas observed on ultrasound as fairly irregular, oval focal abnormalities, sometimes with blurred, uneven external margins. In the present cases, however, the authors observed small labial glands bulging into the oral cavity in the projection of the granulomas mentioned above. Labial glands can also be pushed out by massive fibrosis in the lips observed by the present authors, caused by numerous lip filling procedures, including those using formulations other than the ones based on hyaluronic acid, or by frequent procedures attempting to remove hyaluronic acid or treat any complications associated with the use of hyaluronidase.

Histopathological examination confirmed that the lumps reported by the patients, which were clinically diagnosed as nodules or granulomas, were in fact normal seromucous minor labial glands (Fig. 4).

\section{Discussion}

Lips are skin and muscle folds built of 3 parts: skin, mucosa and the middle part in between (Fig. 5). The labial glands, which are the focus of the present study, were located mainly in the submucosa of the internal part, even though they are usually distributed in the space between the mucosa and the orbicularis oris, and some of them are also found in the muscle layer ${ }^{(4)}$.

The oral cavity and the oropharynx are lined with approx. 600-1000 minor salivary glands of 1-5 mm. The largest number of these glands are found in the lips, buccal mucosa, the tongue and palate, but they are also found in the tonsils, epiglottis and paranasal sinuses ${ }^{(5)}$. The names of the glands are associated with their location: e.g. labial, buccal, lingual. They resemble major salivary glands in histological terms; however, they are much smaller and less branched than their major counterparts. Every minor salivary gland is composed of multiple secretory units and has a short single duct transporting a primarily mucous secretion, but also serous and mixed secretion, directly into the oral cavity. This secretion is necessary for keeping the oral mucosa moisturised and protect it and the teeth against pathogens. Approximately $6-10 \%$ of the total saliva volume comes from these minor glands ${ }^{(4-7)}$.

The lower lip has a higher density, secretion volume and number of active units of minor glands than the upper lip ${ }^{(8)}$.

The success of a lip augmentation procedure depends on good understanding of anatomy, on the technique 


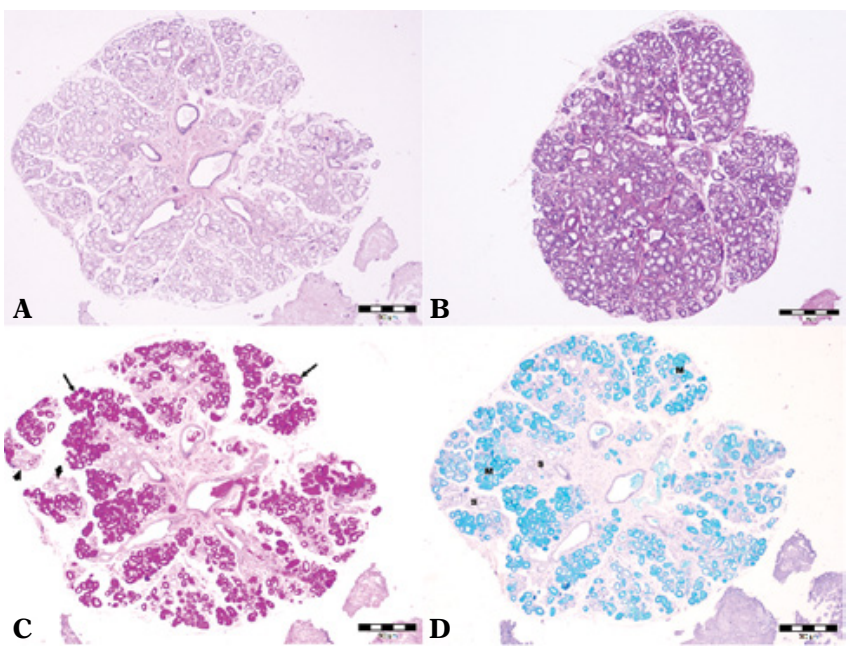

Fig. 4. Labial glands removed as a whole, clinically diagnosed as nodules or granulomas after a filling procedure. A. and B. Haematoxylin and eosin (HE). C. Periodic Acid-Schiff (P.A.S.), mucus-secreting areas (arrows), serous areas (arrow heads). D. Alcian blue, serous cells (S), mucous cells (M)

used, the appropriate selection of filler substance and a sense of aesthetics that allows one to create a natural appearance in the patient ${ }^{(9)}$. This can be achieved by using temporary/degradable/resorbable substances such as collagen or hyaluronic acid or permanent materials. Permanent/non-resorbable fillers include polymethyl methacrylate (PMMA) with or without collagen as a vector, polyacrylamide hydrogel (PAAG), hydroxyethylmethacrylate/ethylmethacrylate (HEMA/EMA) with hyaluronic acid and liquid silicone (polydimethylsiloxane, PDMS $)^{(8)}$. However, none of them meets the criteria for an ideal filler, i.e. a material that primarily achieves the best, long-term effects, causes the lowest number of complications and generates low costs. The main characteristics of an ideal filler should also include nontoxicity, non-carcinogenicity, non-immunogenicity, biocompatibility, plasticity, physical stability and clinical manageability ${ }^{(10)}$. It seems particularly difficult to find such a material for lip augmentation. The use of liquid silicone, which became very popular in the $20^{\text {th }}$ century, is currently forbidden in many parts of the world, such as, for example, in the United States of America or the European Union. Nevertheless, in certain countries of Southern Africa or the Far East, this filler is still popular and accepted ${ }^{(11)}$. However, many individuals are still suffering from late side effects of procedures performed some years ago. The latest guidelines recommend that the use of permanent fillers mentioned above and semi-permanent fillers such as calcium hydroxylapatite (CaHA) and poly-L-lactic acid (PLLA) for lip augmentation be avoided ${ }^{(12)}$. In 2006, the U.S. Food and Drug Administration approved a formulation based on PMMA microspheres suspended in a 3.5\% atelocollagen solution, which is designed for lip procedures, among others $^{(13)}$.

There are also cases of lip augmentation using prohibited substances such as, for example, vitamin A, E and D extracted from gel capsules ${ }^{(14,15)}$.

Hyaluronic acid (HA) is currently the leading filling material used for lip filling and reduction of wrinkles in the area, chiefly due to its temporary nature, degradability, effectiveness, versatility, diversity with over 200 different HA-based formulations present on the market

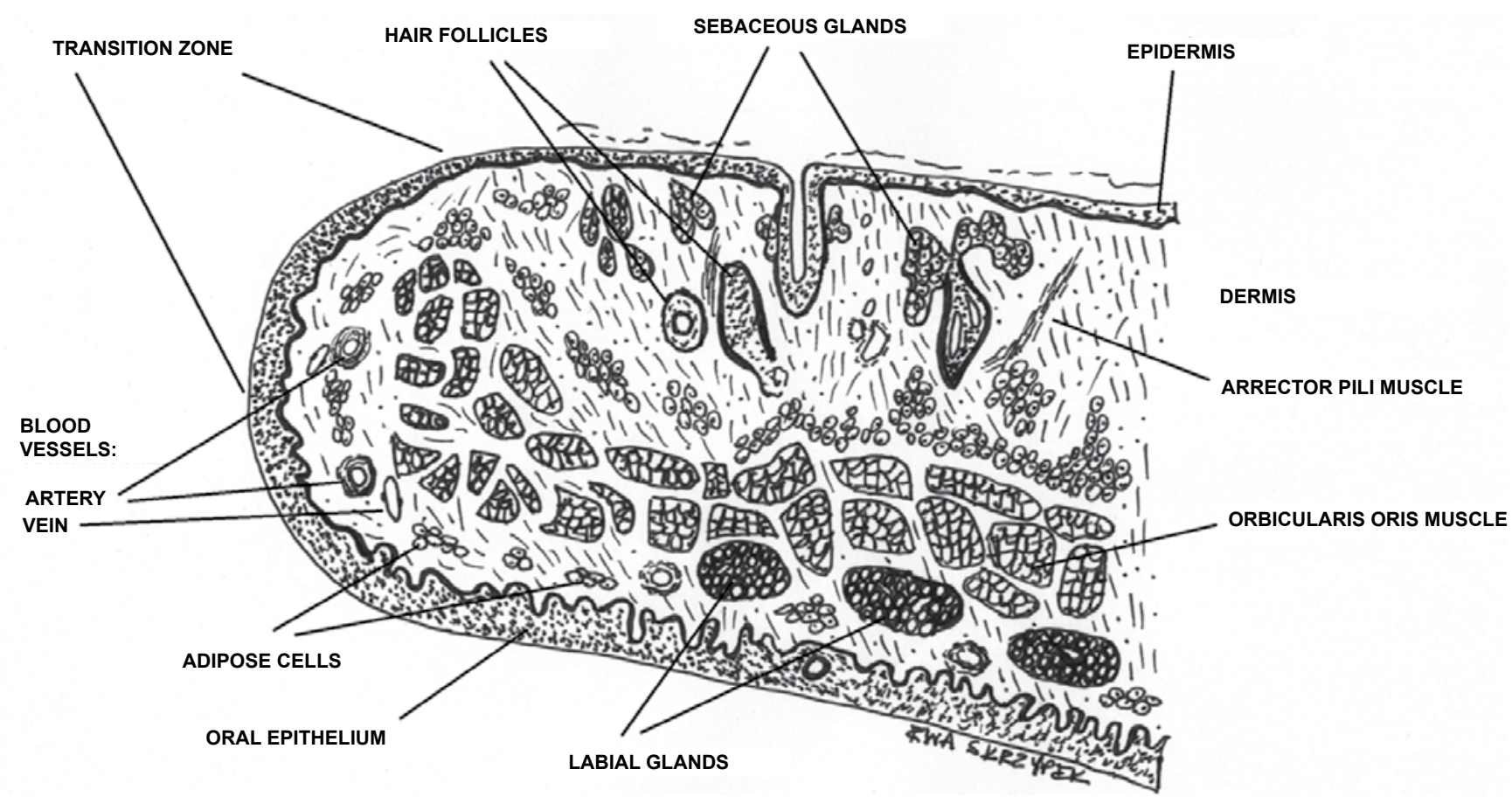

Fig. 5. Lip structure. Drawing by Ewa Skrzypek 
Tab. 2. Hyaluronic acid-based fillers for lip augmentation in adults and lip wrinkle reduction approved by the U.S. Food and Drug Administration

\begin{tabular}{|c|c|c|c|}
\hline Date of approval & Product/manufacturer & Material & Indications \\
\hline $\mathbf{2 0 2 0}$ & Restylane Kysse / Galderma Laboratories, LP & $20 \mathrm{mg} / \mathrm{ml}$; HA, 0.3\% lidocaine & lip augmentation, lip wrinkles \\
\hline $\mathbf{2 0 1 6}$ & Juvéderm Volbella XC / Allergan, Inc & $15 \mathrm{mg} / \mathrm{ml} ; \mathrm{HA}, 0.3 \%$ lidocaine & lip augmentation, lip wrinkles \\
\hline $\mathbf{2 0 1 5}$ & Juvéderm Ultra XC / Allergan, Inc & $24 \mathrm{mg} / \mathrm{ml}$; HA, 0.3\% lidocaine & lip augmentation, lip wrinkles \\
\hline $\mathbf{2 0 1 4}$ & Restylane Silk / Galderma Laboratories, LP & $20 \mathrm{mg} / \mathrm{ml} ; \mathrm{HA}, 0.3 \%$ lidocaine & lip augmentation, lip wrinkles \\
\hline $\mathbf{2 0 1 2}$ & Restylane-L / Galderma Laboratories, LP & $20 \mathrm{mg} / \mathrm{ml} ; \mathrm{HA}, 0.3 \%$ lidocaine & lip augmentation, lip wrinkles \\
\hline $\mathbf{2 0 1 1}$ & Belotero Balance / Merz Aesthetics & $22.5 \mathrm{mg} / \mathrm{ml}$ HA & lip wrinkles \\
\hline $\mathbf{2 0 1 1}$ & Restylane / Galderma Laboratories, LP & lip augmentation, lip wrinkles & \\
\hline HA - hyaluronic acid & &
\end{tabular}

and a favourable safety profile (Tab. 2). Various procedures are used, from the ones which increase general lip volume, define the vermilion border and eliminate asymmetry to the ones which enhance and highlight the lips and reduce the depth of lines and wrinkles around the lips.

Even though such procedures are among the most popular ones, unfortunately, they are constantly associated with a large number of possible complications. The number of such complications has been constantly rising because of the mere increase in the number of procedures and cases when unauthorised individuals perform them. Depending on the time of occurrence, complications can be divided into early and delayed although the latest reports classify them as immediate onset: up to 24 hours after the procedure; early onset: from 24 hours to 4 weeks, and delayed onset: more than 4 weeks following the procedure ${ }^{(16,17)}$. Immediate- and early-onset complications typically occur within hours or days after the procedure, while delayed complications usually develop within weeks or even years after the injection $^{(18)}$. Early-onset complications are well-known and do not differ depending on the filler used. Such complications are usually associated with errors in the injection technique or infections and manifest themselves with prolonged oedema, redness, petechiae, pruritus or pain. The lip area is also susceptible to herpes virus reactivation. Tissue necrosis is a rare but significant earlyonset complication, which is caused by compression, or, more commonly, embolisation of a vessel by the material administered $^{(19)}$. Theoretically, based on research, vision loss is a potential complication of filler injection in this area $^{(20)}$. Delayed-onset complications, on the other hand, caused by product migration, acute or recurrent tissue oedema, infection, and the formation of nodules, granulomas, fistulas and fibrosis can be filler-specific ${ }^{(11)}$.

The most commonly observed side effect in the lip area after filler injection are nodules or granulomas of various sizes. The lips, like the eyelids, have a thin tissue cover; therefore, for instance, the administration of an excessive amount of filler results in lumping or dislocation of the material caused by the movement of active muscles in the area. Apart from that, lips have a high mobility and a characteristically thin mucosa ${ }^{(21)}$. This contributes to the new complication observed by the present authors, which is very significant in diagnostic terms, but has not been reported in the literature to date: small salivary glands within the labial mucosa being pushed out by the filling material.

For this reason, it is not surprising that high frequency ultrasound scanning and histopathological examination play a huge role in the diagnosis of complications after soft tissue filling procedures, particularly suspected abscess, overcorrection, incorrect administration of the filling material, migration, fibrosis, foreign body granulomas, persistent deposit nodules and compression or embolisation of a vessel ${ }^{(22)}$.

High frequency ultrasound makes it possible to precisely identify all lip structures, including small labial glands observed as hypoechoic oval areas located just below the oral mucosa (Fig. 6 and Fig. 7). However, it needs to be emphasised that ultrasound scanning using devices equipped with mechanical, single-element transducers has a high diagnostic value since they allow one to obtain images with higher resolution, resulting in a higher level of detail (Fig. 6) compared to images generated by classic machines (Fig. 7). With such equipment it is easier to differentiate between labial glands and filler deposits or granulomas. In ultrasound images obtained using a 48 $\mathrm{MHz}$ transducer, labial glands are seen as hyperechoic areas with irregular margins and linear hyperechoic reflections correlating with histopathological presentation. The above is also true for the morphology and function of different parts of the orbicularis oris muscle. Ultrasound has been increasingly commonly used in the diagnosis of complications after lip filling procedures since it is non-invasive, safe for the patient, relatively cheap and easily available ${ }^{(3,23)}$.

\section{Conclusions}

The lips are one of the most important parts of facial attractiveness; therefore, reducing the effects of ageing of this particular area and increasing its attractiveness has a prominent place among aesthetic medicine procedures. 


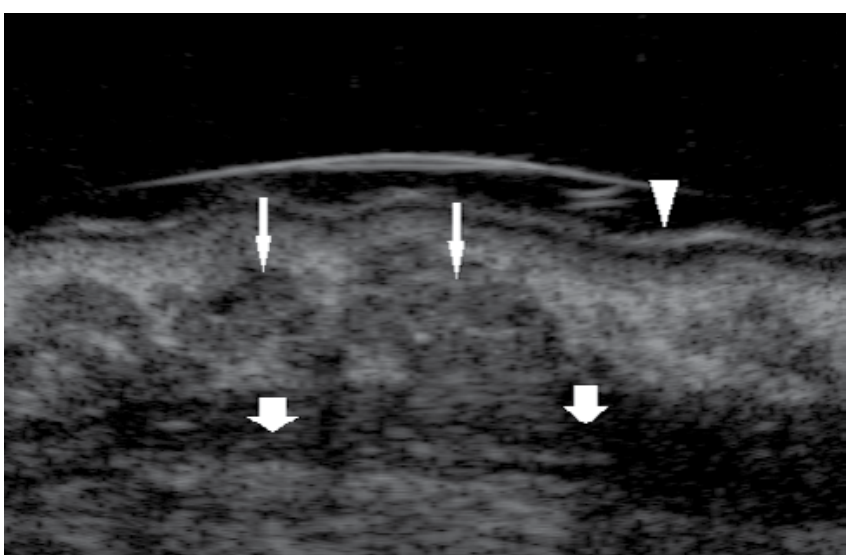

Fig. 6. Ultrasound (DermaMed with $48 \mathrm{MHz}$, transducer) transverse plane of normal labial glands (thin arrows); orbicularis oris (bold arrows); lip mucosa (arrow head)

Despite the fact that primarily hyaluronic acid-based formulations have been used for these procedures in recent times, they are still associated with the risk of numerous complications with uncertain diagnosis. It needs to be emphasised that accurate diagnosis of complications translates into adequate treatment.

An increasing number of women have been seeking medical help having undergone lip filling procedures. They report a complication involving small nodules/ lumps felt from the side of the labial mucosa, which are sometimes uncomfortable during the everyday activity of this area. Sometimes the patients experience a sense of irritation, mild pain or a feeling of piercing and/or burning.

Earlier reports regarding ultrasound differentiation between nodules and granulomas which included histopathological examination made it possible to develop an algorithm for their differentiation using non-invasive ultrasound imaging, since clinical differentiation is rarely possible ${ }^{(3)}$.

The discovery of the actual nature of the small abnormalities described above occurring after lip filling procedures using hyaluronic acid-based formulations, which were previously considered to be only nodules or granulomas, makes the correct diagnosis and treatment possible.

\section{References}

1. Sarnoff DS, Saini R, Gotkin R: Comparison of filling agents for lip augmentation. Aesthetic Surg J 2008; 28: 556-563.

2. Sarnoff DS, Gotkin RH: Six steps to the „perfect” Lip. J Drugs Dermatol 2012; 1: 1081-1088.

3. Mlosek RK, Skrzypek E, Skrzypek DM, Malinowska S: High-frequency ultrasound based differentiation between nodular dermal filler deposits and foreign body granulomas. Skin Res Technol 2018; 24: 417-422.

4. Sumi M, Yamada T, Takagi Y, Nakamura T: MR imaging of labial glands. Am J Neuroradiol 2007; 28: 1552-1556.

5. Myers EN, Ferris RL (ed.): Salivary Gland Disorders, Springer-Verlag Berlin Heidelberg 2007

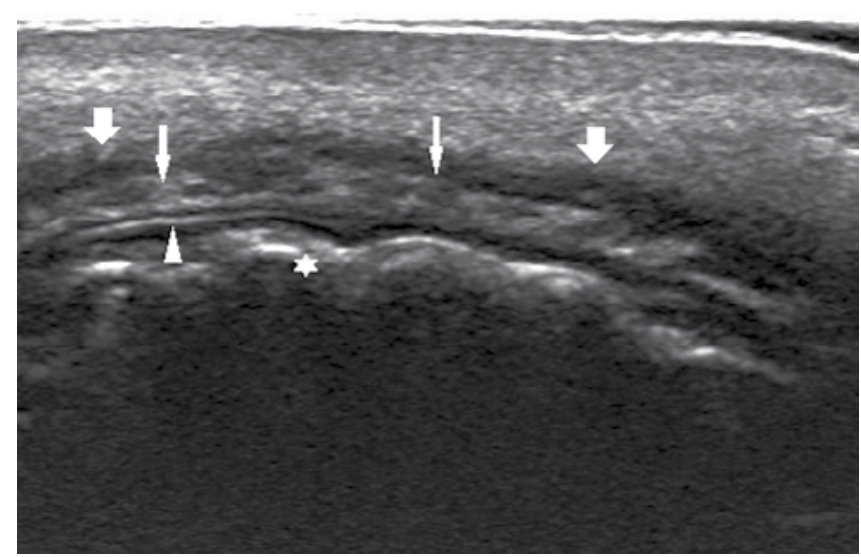

Fig. 7. Ultrasound (Philips Epiq 5) longitudinal plane of normal labial glands (thin arrows); orbicularis oris (bold arrows); lip mucosa (arrow head); teeth (asterisk)

For instance, this excludes the use of hyaluronidase in such cases, which is currently an established part of the algorithm for the treatment of nodules after hyaluronic acid injections. The importance of ultrasound and histopathological examination is also supported for complications after tissue-filling procedures as the only methods that allow one to make the correct diagnosis, and, consequently, apply proper treatment.

As mentioned previously, the above determination of the exact nature of the small lumps in both lips that were reported as a complication and erroneously interpreted clinically as nodules or granulomas is the first such report in the literature, with a huge diagnostic and therapeutic value. This is because a consistent list of complications after tissue filling procedures remains to be developed, with the associated specific diagnostic and therapeutic algorithms.

\section{Conflict of interest}

The authors do not report any financial or personal affiliations to persons or organisations that could adversely affect the content of or claim to have rights to this publication.

6. Eliasson L, Carlén A: An update on minor salivary gland secretions. Eur J Oral Sci 2010; 118: 435-442.

7. Riva A, Puxeddu R, Loy F, Testa-Riva F: Morphofunctional studies on human labial salivary glands. Eur J Morph 2002; 40: 227-233.

8. Gaubenshtok LM, Leont'ev VK: [The quantitative topographic characteristics of the minor salivary glands of the lips] [article in Russian]. Stomatologiia (Mosk) 1990; 69: 28-23.

9. Klein AW: In search of perfect lip. Dermatol Surg 2005; 31: 1599-1603.

10. Scarano A, Puglia F, Cassese R, Mordente I, Amore R, Ferraro G et al.: Hyaluronic acid fillers in lip augmentation procedure: a clini- 
cal andhistological study. J Biol Regul Homeost Agents 2019; 33: S103-S108.

11. Grippaudo FR, Di Girolamo M, Mattei M, Pucci E, Grippaudo C: Diagnosis and management of dermal filler complications in the perioral region. J Cosmet Laser Ther 2014; 16: 246-252.

12. Goldman A, Wollina U: Polymethylmethacrylate-induced nodules of the lips: clinical presentation and management by intralesional neodymium YAG laser therapy. Dermatol Ther 2019; 32: e12755.

13. Dermal fillers approved by the Center for Devices and Radiological Health, https://www.fda.gov/medical-devices/cosmetic-devices/ dermal-fillers-approved-center-devices-and-radiological-health (access: 08.08.2020).

14. Kamouna B, Kazandijeva J, Balabanova M, Dourmishev L, Negentsowa Z, Etugov D et al.: Oil-soluble vitamins: illegal use for lip augmentation. Facial Plast Surg 2014; 30: 635-643.

15. Kamouna B, Litov I, Bardarov E, Broshtilova V, Miteva L, Kazandijeva J: Granuloma formation after oil-soluble vitamin D injection for lip augmentation-case report. J Eur Aacad Dermatol Venereol 2016; 30: 1435-1436.

16. Urdiales-Gálvez F, Delgado NE, Figueiredo V, Lajo-Plaza JV, Mira M, Moreno A et al.: Treatment of soft tissue filler complications: expert consensus recomendations. Aesthetic Plast Surg 2018; 42: 498-510.
17. Gupta A, Miller PJ: Management of lip complications. Facial Plast Surg Clin North Am 2019; 27: 565-570.

18. Abduljabbar MH, Basendwh MA: Complications of hyaluronic acid fillers and their mamagements. J Dermatol Dermatol Surg 2016; 20: $100-106$.

19. Hirsch P, Infanger M, Kraus A: A case of upper lip necrosis after cosmetic filler injection of hyaluronic acid soft-tissue filler - does capillary infarction play a role in the development of vascular compromise, and what are the implications? J Cosmet Dermatol 2020; 19: 1316-1320.

20. Tansatit T, Apinuntrum P, Phetudom T: Cadaveric assessment of lip injections: locating the serious threats. Aesth Plast Surg 2017; 41: $430-440$.

21. Kim JK, Ahn DK, Jeong HS, Suh IS: Treatment algorithm of complications after filler injection: based on wound healing process. J Korean Med Sci 2014; 29: S176-S182.

22. Lefarth FL, Prescher A, Angerstein W: [Comparative ultrasonographic and histomorphologic examination of the lips]. HNO 2014; 62: 879-885.

23. Mlosek K, Słoboda K, Malinowska S: High frequency ultrasound imaging as a potential way of evaluation modality in side effects of lip augmentation - case report. J Cosmet Laser Ther 2019; 21: 203-205. 